\section{AB0699 BENZATHINE PENICILLIN IN TREATMENT OF ULCERS OF BEHCET'S DISEASE}

\section{S. Benamour. ALMS, Casablanca, Morocco}

Background: Prednisone therapy, Thalidomide, azathioprine, anti-TNF-alpha inhibitors are presently used in resistant mucocutaneous lesions of Behçet's Disease (BD). In this study, we used only ambulatory Benzathine Penicillin (BP), without being preceded by high doses of penicillin infusion. This Study confirm the value of $B P$ in the treatment of refractory ulcers of $B D$ and point out a probable role of Streptococcus on the pathogenesis of BD

Objectives: This open Study confirm the value of BP in refractory oral, genital and skin ulcers of BD. We propose to use BP when ulcers of BD are resistant to colchicine $1.5 \mathrm{mg} /$ day and to add BP in the recommendations of EULAR for the treatment of resistant cases of mucocutaneous involvement.

Methods: 15 patients with BD (12 patients fulfilled all criteria for BD) had Giant (mean size of ulcer superior to $1 \mathrm{~cm}$ ), or Multiple oral, genital, skin ulcers received $\mathrm{BP}$ at the dose of 2.4 million units in intramuscular injection; 3 intramuscular injections with an interval of 3 weeks between injection. Colchicine at the dose of $1 \mathrm{mg} /$ day was maintened. Serology of syphilis was negative. Patients had seen 15 days, 3 months and 1 year after the starting treatment.

Results: This study started in 2012, in a private office and comprised 15 patients:9 males; 6 females. Arthritis was observed in 11 patients, uveitis in 6 cases. BP was used in 8 patients with oral ulcers (4 Giants and 4 multiples), Genital ulcers in 3 patients ( 2 multiple and 1 Giant), oral and genital ulcers(2 Multiples) and cutaneous aphtosis in 2 patients 0.2 weeks after the first injection of BP, recovery from ulcers was achieved.BP was administrated a second time with success, in 9 patients who have developed resistant ulcers, after 1 to 3 years of recovery. One patient with panuveitis treated with azathioprine in 2013 and interferon alpha in 2015 and 2017, developed refractory ulcers: Excellent efficacy of BP was observed in this patient.

Conclusions: In this study, we confirm the recovery from refractory oral, genital and cutaneous ulcers (Giant, Multiple) in these 15 patients with BP. We recommend using more frequently $\mathrm{BP}$ and we propose to add $\mathrm{BP}$ in the recommendations of EULAR for the treatment of resistant cases of mucocutaneous involvement because treatment with BP is rather easy in outpatients, it is efficient, it has a low cost and have few side-effects

\section{REFERENCES:}

[1] Benamour S, Bettal S, Alaoui Fz and all: Treatment of Giant aphthosis by IV G Penicillin in Behçet's disease.10 th International Conference on Behçet's disease. Berlin, June 2002

[2] BENAMOUR S: Benzathine Penicillin in treatment of oral and Genital ulcers in Behcet's Disease. 17th International Conference on Behçet's Disease. Matera, 15th-17th September 2016

Disclosure of Interest: None declared

DOI: 10.1136/annrheumdis-2018-eular.2245

\section{AB0700 IS IT FEASIBLE TO WITHDRAW IMMUNOSUPPRESSIVE TREATMENT IN REAL-LIFE PATIENTS WITH ANCA- ASSOCIATED VASCULITIS?}

S. Monti ${ }^{1}, 2$, B. Vitolo ${ }^{1}$, S. Balduzzi ${ }^{1}$, M. Felicetti ${ }^{3}$, R. Padoan ${ }^{3}$, A. Berti ${ }^{4}$, M. Bond $^{5}$, L. Quartuccio ${ }^{5}$, G. Paolazzi ${ }^{4}$, F. Schiavon ${ }^{3}$, C. Montecucco ${ }^{1}$, R. Caporali ${ }^{1}$. ${ }^{1}$ Rheumatology, IRCCS Policlinico S. Matteo Foundation; ${ }^{2}$ University of Pavia, PhD in Experimental Medicine, Pavia; ${ }^{3}$ Rheumatology, University of Padova, Padova; ${ }^{4}$ Rheumatology, Santa Chiara Hospital, Trento; ${ }^{5}$ Rheumatology, University Hospital S. Maria della Misericordia, Udine, Italy

Background: The optimal duration of remission-maintenance treatment in ANCA-associated vasculitis (AAV) is still unknown, ${ }^{1}$ with International recommendations suggesting to maintain it for at least 24 months. Data supporting the correct balance between relapse risk, treatment-related adverse events and damage are highly needed.

Objectives: To analyse the frequency and predictors of withdrawal of remissionmaintenance immunosuppressive drugs (IS) in a large real-life cohort of AAV patients.

Methods: Clinical records of patients with granulomatosis with polyangiitis (GPA) or microscopic polyangiitis (MPA) were retrospectively analysed from a multicentric cohort. We divided the cohort into group A (withdrawal) and group B (ongoing).

Results: We included 185 patients. Forty-one patients $(22 \%)$ had withdrawn IS at the end of follow-up (median 73.5 months, IQR 54-115). Concomitant glucocorticoids were stopped in $33 \%$. There were no significant differences in AAV subtype,
ANCA pattern, age at diagnosis, diagnostic delay or comorbidities rates between group A and group B (table 1). Disease onset with pulmonary involvement was significantly more frequent in patients in whom IS was maintained $(69.7 \%$ vs $45 \%$; $\mathrm{p}=0.004$ ). Figure 1 . Disease activity at onset, but not during follow-up, was significantly higher in patients from group B (ongoing): median BVAS 18 (12-24) vs 15 $(8-20) p=0.02$. The type of IS used for remission induction did not differ between the two groups. Maintenance treatment with Rituximab (RTX) was associated with higher probability of discontinuing IS by the end of follow-up (21\% vs $7.6 \%$; $p=0.02$ ). There was no difference in the number of major relapses between the two groups. Safety profile was equally good in both groups, except for a higher number of infections over the course of disease in patients who withdrew IS (5\% vs 0 ). Vasculitis damage index (VDI) was comparable between the two groups.

Abstract AB0700 - Table 1. Clinical characteristics and outcome: comparison between IS withdrawal group (A) and ongoing group (B).

\begin{tabular}{lccc}
\hline & $\begin{array}{c}\text { Group A } \\
\text { (withdraw) } \\
\mathrm{n}=41\end{array}$ & $\begin{array}{c}\text { Group B } \\
\text { (ongoing) } \\
\mathrm{n}=144\end{array}$ & $\mathrm{p}$ \\
\hline Sex: & $18(43.9 \%)$ & $67(46.5 \%)$ & 0.769 \\
Male, N (\%) & $4(2-11.75)$ & $3.5(1-8)$ & 0.551 \\
Diagnostic delay, months, median (IQR) & $53.85 \pm 16.30$ & $54.41 \pm 15.39$ & 0.847 \\
Age at diagnosis (mean+SD) & $73.5(54-115)$ & $66(36.5-109.5)$ & 0.315 \\
Mean follow up duration months, median & & & \\
(IQR) & $32 / 41(78 \%)$ & $112 / 144(77.8 \%)$ & 0.976 \\
AAV subtypes N(\%) & $9 / 41(21.9 \%)$ & $29 / 144(20.1 \%)$ & 0.801 \\
GPA & $10 / 41(24.4 \%)$ & $47 / 143(32.9 \%)$ & 0.302 \\
MPA & $19 / 41(46.3 \%)$ & $78 / 143(54.5 \%)$ & 0.355 \\
p-ANCA (MPO) N(\%) & $0(0-1)$ & $0(0-1.25)$ & 0.695 \\
c-ANCA (PR3) N(\%) & $6 / 41(14.6 \%)$ & $11 / 135(8.1 \%)$ & 0.220 \\
Number of major flares, median (IQR) & $2 / 40(5 \%)$ & $0 / 134(0 \%)$ & 0.009 \\
Safety: cancer & & & \\
Safety: infections: & $0(0-1)$ & $0(0.1)$ & 0.454 \\
$>3$, N(\%) & $3(2-4.75)$ & $3(2-4)$ & 0.773 \\
Number of hospitalisations, median (IQR) & & & \\
VDI last follow-up & & &
\end{tabular}

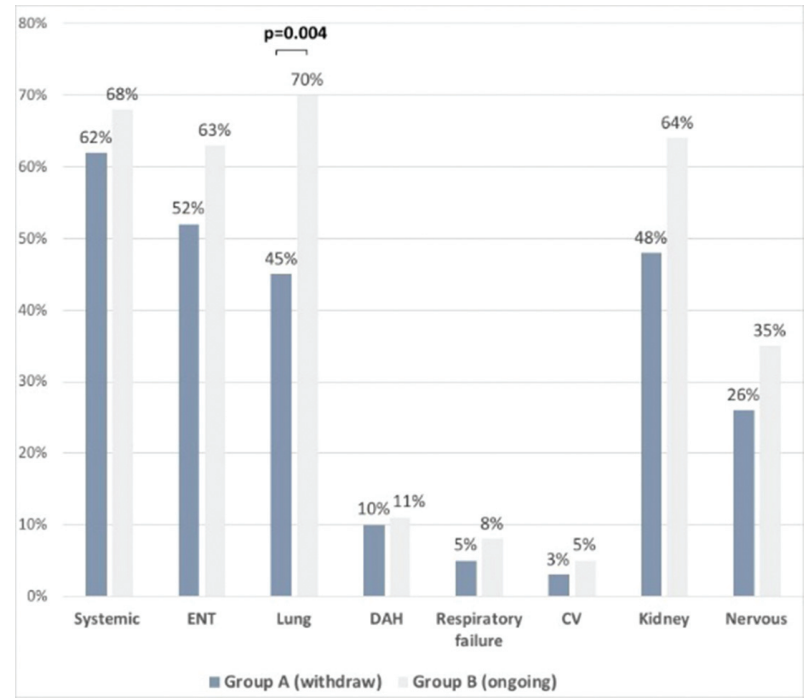

Abstract AB0700 - Figure

Conclusions: IS withdrawal was observed in a minority of real-life patients with AAV. Drug-free remission was negatively associated with pulmonary involvement and higher BVAS at disease onset. Remission maintenance with RTX was associated with a higher frequency of drug withdrawal at the end of follow-up.

\section{REFERENCE:}

[1] Karras, et al. Randomised controlled trial of prolonged treatment in the remission phase of ANCA-associated vasculitis. Ann Rheum Dis 2017;76:1662-68

Disclosure of Interest: None declared DOI: 10.1136/annrheumdis-2018-eular.4838 